\title{
A rare cause of crepitus
}

\author{
H M Paterson, N Mamode
}

A 52-year-old man presented to Accident and Emergency complaining of pain in his right upper chest and right arm which he attributed to falling out of bed approximately 36 hours earlier. On examination he was apyrexial, normotensive and mildly tachypnoeic. His right upper arm and chest were swollen and crepitant to palpation. There were no open wounds. Chest examination was otherwise unremarkable. His chest radiograph (figure 1) showed no evidence of lung collapse. The lucent areas along fascial planes and rib fractures in the upper thorax were thought to confirm that a resolving pneumothorax was responsible for the apparent subcutaneous emphysema. He was admitted for observation but within an hour the appearance of his right arm had deteriorated dramatically (figure 2 ).

Department of Surgery, Ayr Hospital, Ayr KA6 6DX, UK H M Paterson

N Mamode

Correspondence to $\mathrm{Dr} \mathrm{H} \mathrm{M}$ Paterson, 2 Lomondside Avenue, Clarkston, Glasgow G76 7UL, UK

Accepted 25 June 1997

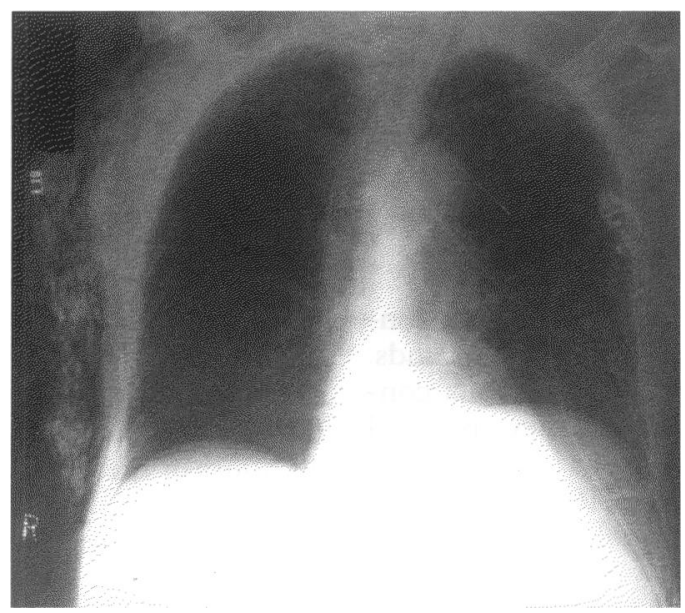

Figure 1 Chest X-ray

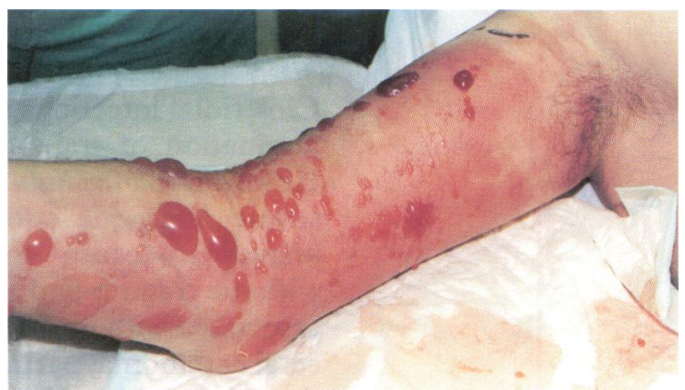

Figure 2 Right arm of the patient

\section{Questions}

1 What is the diagnosis?

2 What two therapeutic steps would you institute at this stage? 


\section{Answers}

QUESTION 1

The diagnosis is spontaneous (non-traumatic) gas gangrene and the swelling, mottling and dermal bullae shown in figure 2 are classical appearances. The causative organism in the majority of cases is Clostridium septicum. ${ }^{1}$

QUESTION 2

Spontaneous gas gangrene is a fulminant and frequently fatal condition. Successful management of clostridial myonecrosis depends on rapid recognition followed by immediate surgical debridement and high doses of intravenous penicillin ( $2.4 \mathrm{~g} \mathrm{4-6}$ hourly). The use of hyperbaric oxygen has been shown to reduce mortality and morbidity in some cases but has not gained widespread acceptance in the UK and at present plays only an adjunctive role. In this case, despite repeated debridement (including disarticulation of the arm), antibiotics and aggressive supportive therapy, the patient died within 48 hours of admission.

\section{Discussion}

Clostridial infections typically result from contamination of traumatic or surgical wounds. However, Clostridium septicum infections constitute a unique subgroup as they are well known to cause spontaneous myonecrosis without trauma and in over $80 \%$ of cases are associated with underlying malignancy. ${ }^{2}$ Colorectal carcinoma, particularly of the right side of the colon, is identified in approximately $50 \%$ of these cases and haematological malignancy identified in the remaining $50 \% .^{3}$ It has been postulated that mucosal ulceration of the large

1 Kornbluth AA, Danzig JB, Bernstein LH. Clostridium septicum infection and associated malignancy: report of two cases and review of the literature. Medicine 1989;68:30-7. 2 Alpern RJ, Dowell VR. Clostridium septicum infections and malignancy. $\mathscr{F} A M A$ 1969;209:385-8.

3 Larson CM, Bubrick MP, Jacobs DM, West MA. Malignancy, mortality, and medicosurgical management of Clostridium septicum infection. Surgery 1995;118:592-8.

\section{Learning points}

- consider spontaneous gas gangrene if there is pain out of proportion to signs and especially if there is swelling and crepitus in the absence of wounds

- rapid recognition followed by prompt and aggressive debridement is essential

- survivors should be fully investigated for concurrent malignancy

bowel is the common portal for infection in these conditions and that since the organism has not been identified in the bowel flora of healthy individuals, it may represent an opportunistic infection. ${ }^{4}$ Why this particular clostridial species should be so strongly linked with these conditions and not with other causes of bowel ulceration (eg, Crohn's disease) is unknown.

Symptoms and signs of spontaneous gas gangrene present in equal measure on the trunk by local invasion or on extremities by metastatic spread. Mortality is greater in the latter group and is reported to be between $60-100 \%$ for all cases, often occurring within 48 hours of presentation. ${ }^{5}$

\section{Final diagnosis}

Spontaneous (non-traumatic) gas gangrene.

Keywords: Clostridium septicum; spontaneous gas gangrene

4 Koransky JR, Stargel MD, Dowell VR. Clostridium septicum bacteremia: its clinical significance. Am $\mathcal{F} M e d$ 1979;66:636.

5 Stevens DL, Musher DM, Watson DA, et al. Spontaneous, non-traumatic gangrene due to Clostridium septicum. Rev Infect Dis 1990;12:286-96. 